\section{Silencing of S100A4, a metastasis-associated protein, inhibits retinal neovascularization via the downregulation of BDNF in oxygen- induced ischaemic retinopathy}

G Cheng, T He and Y Xing

\begin{abstract}
Background To investigate the underlying mechanism of S100A4 function and whether it has a role in retinal neovascularization (RNV) in a mouse model of oxygen-induced retinopathy (OIR).

Methods Retinas from a mouse model of OIR were treated with and without an intravitreous injection of adenoviral-S100A4-RNAi or adenoviral green fluorescence protein (GFP) at postnatal day 12 (P12). At P17, the efficacy of adenoviral gene transfer was assessed using fluorescence microscopy and western blot analysis. RNV was evaluated by wholemount immunofluorescence staining of the mouse retina and by counting the number of pre-retinal neovascular cells. Protein and mRNA expression levels of S100A4, brainderived growth factor (BDNF), and vascular endothelial growth factor (VEGF) were measured using western blot analysis and real-time PCR.

Results Retinal S100A4 levels were positively correlated with the progression of RNV. In the OIR-S100A4-RNAi group, both protein and mRNA expression levels of S100A4 in the retina significantly decreased at P17 compared with those in the OIR group. Ad-S100A4-RNAi transfer was clearly demonstrated by GFP fluorescence in many layers of the retina 5 days after the Ad-S100A4-RNAi transfer. Whole-mount immunofluorescence staining of the retina and quantification of the pre-retinal neovascular cells demonstrated that RNV was significantly inhibited. Meanwhile, the levels of the transcription and translation of BDNF,
\end{abstract}

VEGF, and hypoxia-inducible factor- $1 \alpha$ (HIF-1 $\alpha$ ) significantly decreased in the OIRS100A4-RNAi group.

Conclusions Ad-S100A4-RNAi transfer ameliorates RNV. The related mechanism may involve silencing S100A4 to decrease the activation of BDNF, which downregulates VEGF expression via HIF-1 $\alpha$. This finding could provide a new therapeutic target for the treatment of ocular neovascularization diseases.

Eye (2016) 30, 877-887; doi:10.1038/eye.2016.43; published online 18 March 2016

Introduction

Retinal neovascularization (RNV) is the common pathophysiological basis of all retinal vascular diseases and is a complex process and major cause of blindness in developed countries. ${ }^{1,2}$ Ischaemia-induced retinopathy is clearly a primary aetiological factor associated with RNV. ${ }^{3}$ Laser photocoagulation is usually clinically applied to inhibit the development of RNV and has shown some therapeutic effects. ${ }^{4}$ However, laser treatment only delays the progression of lesions and may potentially further damage retinal tissue, leading to the side effects including diminished night vision, reduced peripheral vision, and decreased visual acuity. ${ }^{5}$ During the last decade, there has been a noticeable improvement in the understanding of the underlying molecular and cellular mechanisms of RNV. This has been essential for
Eye Center, Renmin Hospital of Wuhan University, Wuhan, China

Correspondence: $\mathrm{T}$ He or $\mathrm{Y}$ Xing, Eye Center, Renmin Hospital of Wuhan University, 238 Jiefang Road, Wuhan 430060, China

Tel: +86 2788041911

81218 or +8627 8804191182038 ; Fax: +86 2788042292. E-mail: hetao0216@gmail. com or yiqiaoxing1111@ gmail.com

Received: 26 August 2015 Accepted in revised form:

4 February 2016

Published online:

18 March 2016 
the identification and introduction of new therapeutic interventions designed to suppress this process. Antiangiogenic strategies involving intravitreal injections of anti-vascular endothelial growth factor (VEGF) and the administration of anti-PDGF agents have emerged as valuable new therapies for ocular neovascularization. ${ }^{6,7}$ Therefore, a new challenge has arisen to identify the other major factors involved in

RNV and to develop new treatments. In searching for potential targets to treat oxygen-induced ischaemic retinopathy, the protein S100A4 attracted our attention.

S100A4, a calcium-binding protein, is localised in the nucleus, cytoplasm, and extracellular space and possesses a wide range of biological functions, such as the regulation of angiogenesis, cell survival, motility, and invasion. ${ }^{8}$ In previous works, S100A4 was established as a marker and mediator of metastatic disease. One of the mechanisms responsible for its metastasis-promoting effects is its function as a potent stimulator of angiogenesis, which is based on observations in transgenic mice. ${ }^{9-11}$ However, the role and mechanism of S100A4 in RNV have not been characterized.

In the present study, adenoviral-mediated S100A4 RNAi transfer was used to silence S100A4 gene expression in the retinas of an oxygen-induced retinopathy (OIR) mouse model, and for the first time, we showed that the silencing of S100A4 gene expression significantly ameliorated RNV.

\section{Materials and methods}

\section{Ethics statement}

This study was carried out in strict accordance with the recommendations in the Guide for the Care and Use of Laboratory Animals of Wuhan University. The protocol was approved by the Committee on the Ethics of Animal Experiments of Wuhan University. All surgeries were performed using sodium pentobarbital anaesthesia, and all efforts were made to minimise suffering.

\section{Recombinant adenoviral vector construction}

Small interfering RNA (siRNA) is a non-protein coding string of nucleotides that originates from invading viruses, transgene inverted repeats, centromeres, transposons, and other receptive sequences. siRNA is thought to be an RNAbased immune mechanism that fights off nucleic acid invaders and maintains genome stability. ${ }^{12}$ In our study, two recombinant adenoviral vectors based on the GV120 vector (Figure 1a; purchased from GeneChem Co, Ltd, Shanghai, China) were constructed to silence the mouse S100A4 gene (Ad-S100A4-RNAi) and to express the green fluorescence protein gene (Ad-GFP; negative control). The target sequence is 5'-TGA GCA ACT TGG ACA GCA A-3', and the negative control sequence is $5^{\prime}$-TTC TCC GAA CGT GTC ACG T-3'. The stock solutions of Ad-S100A4RNAi and Ad-GFP were $2 \times 10^{12}$ PFU per ml. A working solution was prepared to make $1 \mu \mathrm{l}$ of vehicle containing $\sim 1.0 \times 10^{9}$ PFU.

\section{Mouse model of OIR}

Pregnant female C57BL/6J mice were provided by the Laboratory Animal Center of Wuhan University. All experiments were conducted in accordance with the Association for Research in Vision and Ophthalmology Resolution on the Care and Use of Laboratory Animals. The mouse model of OIR exposes 7-day-old (P7) mouse pups to $75 \pm 2 \%$ oxygen for 5 days (until P12). This results in the regression and vaso-obliteration of the central retinal vasculature. The mice are then returned to room air until P17, during which time neovessels form at the junction between the vascularises and non-vascularises retina. As described above, this model is useful in delineating the mechanisms involved in initial vessel loss (P7-P12), vascular regrowth (P12-P17), neovessel formation (P14-P17), and neovascular regression (P17-P25). ${ }^{13}$

The experimental mice were randomly divided into four groups: the normal control group (the control group), the untreated OIR group (OIR group), the OIR treated with Ad-S100A4-RNAi group (the OIR-S100A4-RNAi group), and the OIR treated with Ad-GFP group (the OIR-GFP group). In the control group, newborn mouse pups were maintained in room air from P0 to P17. In the OIR group, OIR was induced in C57BL/6J mouse pups from P7 to P17. In the OIR-S100A4-RNAi group and OIR-GFP group, the OIR induction protocol was the same as in the OIR group. Then, the mice were given an intravitreal injection of $1 \mu \mathrm{l}$ of vehicle containing $\sim 1.0 \times 10^{9}$ PFU of Ad-S100A4-RNAi or Ad-GFP at P12 and returned to normoxia for the next 5 days. Mice in all four groups were killed at P17, and retinas were collected for biochemical assays and morphological studies.

\section{Intravitreous injection of recombinant adenoviral vectors in mice}

A Harvard pump microinjection apparatus and pulled glass micropipettes were used for precise intravitreous injections. ${ }^{14}$ After anaesthetisation with $2 \%$ isoflurane, one drop of $0.5 \%$ alcaine was applied to the mouse eyes as a topical anaesthetic before intravitreous injection. The mouse was positioned properly, and the superior nasal region of the eye was exposed. Each micropipette was calibrated to deliver $1 \mu$ l of vehicle containing $\sim 1.0 \times 10^{9}$ 


\section{a}
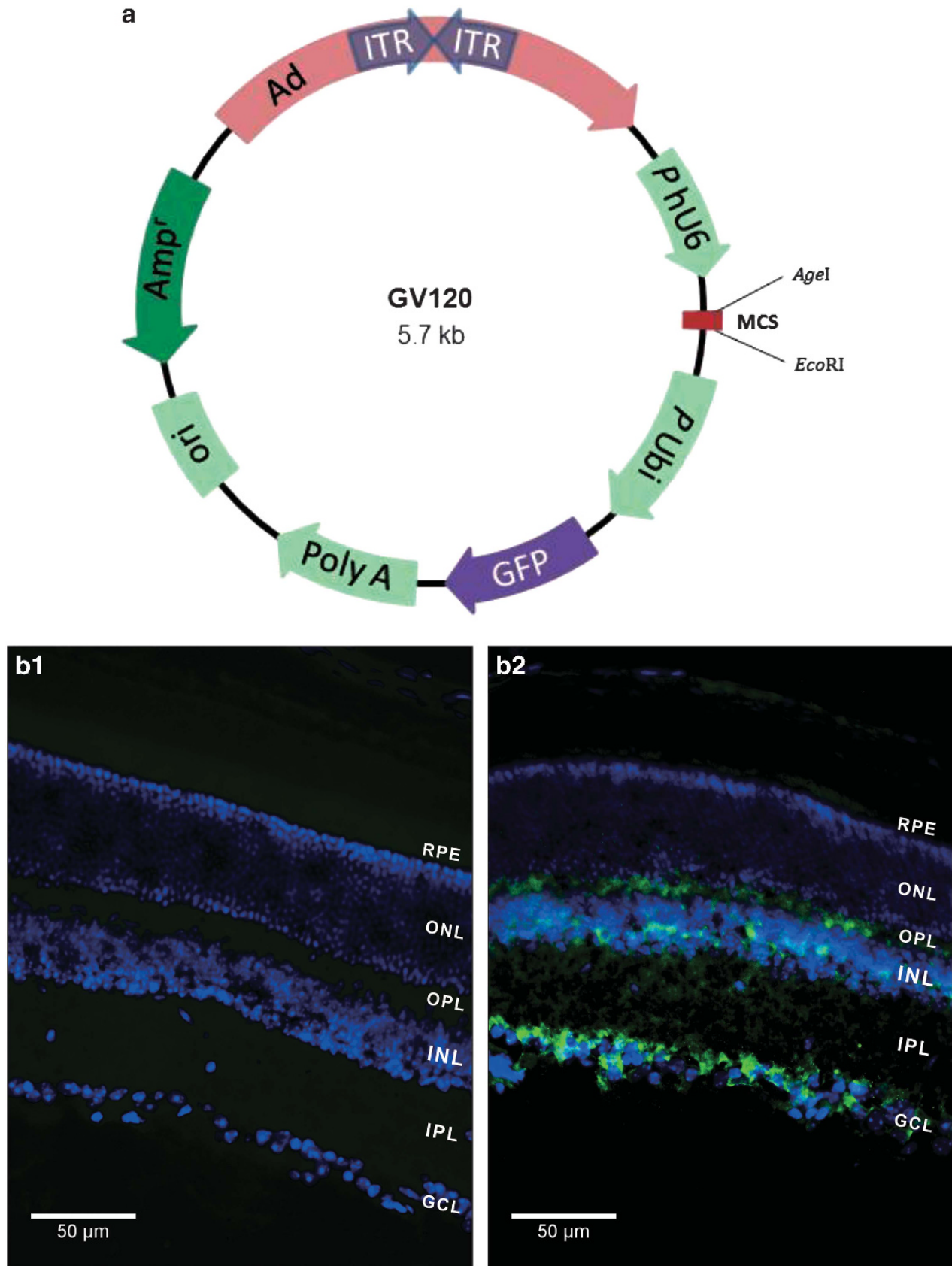

Figure 1 Schematic representation of the GV120 plasmid and the efficacy of Ad-S100A4-RNAi transfer into the mouse retina. (a) ITR, inverted terminal repeat; PhU6, human U6 promoter; MCS, multiple cloning site; AgeI, restriction enzyme; EcoRI, restriction enzyme; PUbi, ubiquitin promoter; GFP, green fluorescent protein; Poly A, SV40 polyadenylation signal; Ori, Escherichia coli origin of replication; Amp, ampicillin resistance sequence. Ad: mouse S100A4 RNAi coding sequence. (b1, b2) The Ad-S100A4-RNAi transferred into the retina of a P12 C57BL/6J mouse 5 days after the intravitreal injection of $\sim 1.0 \times 10^{9}$ plaque-forming units of Ad-S100A4-RNAi. Retinas were obtained from the control group and OIR-S100A4-RNAi group mice for cryosection, which were immunostained with an antibody for GFP and observed using fluorescence microscopy. Fluorescence of GFP can hardly be seen in retina cryosections from the control group (b1) (treated with nothing) but can be easily observed in the ganglion cell layer (GCL), inner plexiform layer (IPL), inner nuclear layer (INL), and the outer plexiform layer (OPL), and less easily observed in the outer nuclear layer (ONL) of retinas from the OIRS100A4-RNAi group (b2) (treated with Ad-S100A4-RNAi). Images b1 and b2 were taken at $400 \times$ magnification for optimal comparison; scale bars $=50 \mu \mathrm{m}$. 
PFU of Ad-S100A4-RNAi or Ad-GFP into the posterior chamber. After the intravitreous injection, we applied ophthalmic Tobrex ointment to the mouse eye to prevent infection. ${ }^{15}$

\section{Fluorescence microscopy after intravitreal injection}

To examine the transfection effect of Ad-S100A4-RNAi on the retina, 5 days after intravitreal injection of the Ad-S100A4-RNAi or Ad-GFP vector, the mice were killed to collect the retinas for cryosections. Specimens were processed as described previously. ${ }^{16}$ The eyecups were embedded in OCT compound (Tissue-Tek, Sakura, Torrance, CA, USA) and were vertically sectioned with a $12-\mu \mathrm{m}$ thickness using a Leica CM1900 cryostat (Leica, Wetzlar, Germany). The sections were mounted on polylysine-coated slides. The sections of the midperipheral retina $(0.6 \mathrm{~mm}$ from the optic disk) were immunostained with an anti-GFP antibody (ab190203, Abcam, Cambridge, UK) and then used for fluorescence microscopy.

\section{Quantification of RNV by whole-mount immunofluorescence staining of the mouse retina}

Whole-mount retinal staining was performed following the previously described protocol. ${ }^{17}$ Enucleated eyes were fixed in $4 \%$ PFA for $1 \mathrm{~h}$. The anterior segment of the eye and vitreous humour were removed. The retinas were dissected from the sclera and flattened on a glass slide with four radial incisions reaching $\sim 2 / 3$ of the radius of the retina to create a 'petal' shape. Then, the retinas were gently rinsed in PBS for $5 \mathrm{~min}$ and blocked in buffer containing $5 \%$ bovine serum albumin (BSA) and $0.2 \%$ Triton X-100 in PBS for $1.5 \mathrm{~h}$ at room temperature. Retinas were incubated for 3 days with Griffonia simplicifolia isolectin B4 conjugated to Alexa Fluor 594 (1 : 200, Invitrogen, Carlsbad, CA, USA, 121413) and mounted in anti-fade mounting medium. Images were captured using a fluorescent microscope.

Adobe Photoshop CS3 (San Jose, CA, USA) was used for the quantification of vaso-obliteration and NV, which was processed as previously described. ${ }^{13}$

\section{Quantitative assessment of RNV by the counting of pre-retinal neovascular cells}

P17 mice were anaesthetised and killed by perfusion with $4 \%$ PFA. Eyes were enucleated, and they then received additional fixation in $4 \%$ PFA for $24 \mathrm{~h}$ at room temperature and were embedded in paraffin. Serial sections $(6 \mu \mathrm{m})$ of whole eyes were sagittally cut through the cornea and parallel to the optic nerve and stained with periodic acid-Schiff and haematoxylin. ${ }^{18}$ Only sections through the optic nerve were selected. Nuclei from new vessels and vessel profiles could be distinguished from other structures in the retina and counted in cross-section using light microscopy.

\section{Western blot analysis}

Western blotting was performed according to the standard protocols. ${ }^{19}$ Retinas from P17 mice were prepared for protein extraction; then, the total protein concentration was measured using a BCA Protein Quantification Kit (Abcam, ab102536) according to the manufacturer's recommendations. Proteins were separated by $12 \%$ SDS-PAGE and transferred to polyvinylidene fluoride membranes.

The membrane was blocked with $5 \%$ non-fat milk or $5 \%$ BSA (depending on the antibody) in Tris-buffered saline containing $0.1 \%$ Tween 20 (TBST) for $2 \mathrm{~h}$ at room temperature. After washing with TBST for $10 \mathrm{~min}$ (three times), the membrane was incubated with primary antibody diluted in the blocking solution overnight at $4{ }^{\circ} \mathrm{C}$. The primary antibodies used in our study were the following: rabbit polyclonal antibody against S100A4 (S100A4, 1 : 100, Abcam, ab27957), rabbit monoclonal antibody against brain-derived neurotrophic factor (BDNF, 1 : 1000, Santa Cruz, Dallas, TX, USA, sc-546), rabbit monoclonal antibody against VEGF $(1: 1000$, Santa Cruz, sc-507), and mouse monoclonal antibody against $\beta$-actin ( $\beta$-actin, $1: 10000$, Abcam, ab6276). The membrane was washed thoroughly with TBST for $10 \mathrm{~min}$ (three times), and then incubated with HRP-conjugated goat anti-rabbit or goat anti-mouse IgG (1 : 1000, PTGLab, Rosemont, IL, USA) for $2 \mathrm{~h}$ at room temperature. The immunoreactive bands were developed with enhanced chemiluminescence and detected by photographic film. To estimate the molecular weight of proteins, a prestained marker (Fermentas, Baltimore, MD, USA) was used. Protein levels were quantified using densitometry via Quantity One software (Bio-Rad Laboratory, Hercules, CA, USA) for analysis.

\section{Real-time PCR}

Total RNA was extracted from the frozen retina tissue using Trizol reagent (Invitrogen, 15596-026) according to the manufacturer's instructions. cDNA synthesis was conducted according to the RNA PCR core kit (Invitrogen, N8080143) protocol. $\beta$-Actin was used as a normalising control. The primer sequences were S100A4: $5^{\prime}-$ GTG TCC ACC TTC CAC AAA TAC TCA-3' (forward) and 5'-ACT TCA TTG TCC CTG TTG CTG TC-3' (reverse); BDNF: 5'-GAG TGG GTC ACA GCG GCA GAT AA-3' (forward) and 5'-ATA CGA TTG GGT AGT TCG GCA TT-3' (reverse); VEGF: 5'-CAG GCT GCT GTA ACG 
ATG AA-3' (forward) and 5'-TTT GAC CCT TTC CCT TTC CT-3 (reverse); hypoxia-inducible factor- $1 \alpha$ (HIF- $1 \alpha)$ : 5'-TTC TCC AAG CCC TCC AAG TAT GA-3' (forward) and 5'-ATG CCT TAG CAG TGG TCG TTT CT-3' (reverse); and $\beta$-actin: 5'-CTG AGA GGG AAA TCG TGC GT-3' (forward) and 5'-CCA CAG GAT TCC ATA CCC AAG A-3' (reverse). The PCR reaction was performed in a volume of $20 \mu \mathrm{l}$ using SYBR green mix (Toyobo, Shanghai, China) on the Rotor-Gene 3000 Real-time PCR instrument (Corbett Research, Sydney, New South Wales, Australia). The thermal cycling programme consisted of one cycle of $50{ }^{\circ} \mathrm{C}$ for $2 \mathrm{~min}$, and then $95^{\circ} \mathrm{C}$ for $10 \mathrm{~min}$, followed by 40 cycles of $95^{\circ} \mathrm{C}$ for $30 \mathrm{~s}$ and $60{ }^{\circ} \mathrm{C}$ for $30 \mathrm{~s}$. Relative quantification of the gene expression was performed using the $2^{(-\Delta \Delta C T)}$ method. ${ }^{20}$

\section{Statistical analysis}

All values are given as the mean \pm SD. Group differences were analysed with one-way ANOVA followed by Bonferroni post-test. $P$-values $<0.05$ were considered statistically significant.

\section{Results}

Efficacy of Ad-S100A4-RNAi transfer into the mouse retina

Fluorescence microscopy was used to estimate the transfection effect of Ad-S100A4-RNAi in the mouse retina. Fluorescence microscopy of retinal sections revealed that Ad-S100A4-RNAi was clearly present in the ganglion cell layer (GCL), inner plexiform layer (IPL), inner nuclear layer (INL), outer plexiform layer (OPL), and less strongly in the outer nuclear layer (ONL) of the retina. Mouse retinas treated with intravitreous injections of Ad-GFP showed the same amount of fluorescence in the GCL, IPL, INL, OPL, and ONL as retinas transfected with Ad-S100A4-RNAi. In the control group without intravitreous injection, no positive amount of fluorescence was detected (Figure 1b1 and b2).

\section{Change in S100A4 protein and mRNA levels after Ad-S100A4-RNAi transfer into the mouse retina}

Western blot analysis showed that the S100A4 protein levels were substantially reduced in the OIR-S100A4RNAi group $(0.26 \pm 0.05)$ compared with those in the control group $(1.05 \pm 0.19, t=3.61, P<0.05)$, OIR group $(2.76 \pm 0.47, t=11.49, P<0.05)$, and OIR-GFP group $(2.35 \pm 0.15, t=9.59, P<0.05)$. (Figures $2 \mathrm{a}$ and $\mathrm{b}$ ).

Real-time PCR assays further confirmed that the mRNA expression levels of S100A4 decreased in the OIR-S100A4-RNAi group $(0.18 \pm 0.08)$ compared with those in the control group $(0.62 \pm 0.05, t=9.02, P<0.05)$, OIR group $(1, t=16.91, P<0.05)$, and OIR-GFP group $(0.92 \pm 0.06, t=15.15, P<0.05$; Figure $2 \mathrm{c})$. The results suggested that Ad-S100A4-RNAi transfer can efficiently silence S100A4 gene expression at both the levels of transcription and translation.

\section{Effects of Ad-S100A4-RNAi transfer on oxygen-induced ischaemic RNV}

To assess whether Ad-S100A4-RNAi transfer suppresses oxygen-induced RNV, we examined the retinal vasculature in all four groups using immunofluorescence staining of the vasculature on retinal flat mounts at P17. The results revealed a significant neovascularization in the whole-mounted retinas from the OIR group and OIR-GFP group (Figures $3 c$ and d). In contrast, less severe neovascular tufts and vaso-obliteration areas were developed in retinas from the OIR-S100A4-RNAi group (Figure 3b). Retinas from control group mice showed no neovascularization and vaso-obliteration (Figure 3a). The extent of RNV was quantified by measuring the area of neovascular tufts in retina whole mounts using Image J 1.48V software (NIH, Bethesda, MD, USA), which showed that the retinas from the OIR-S100A4RNAi group had significantly smaller retinal neovascular tufts area than those in the OIR group $(t=8.78, P<0.05)$ and the OIR-GFP group $(t=10.24$, $P<0.05$ ), respectively (Figure $3 e$ ). In addition, to assess whether Ad-S100A4-RNAi transfer ameliorates the extent of vaso-obliteration in OIR, the vaso-obliteration areas in the central retina at P17 were measured by the same software, as previously described. In the OIRS100A4-RNAi group, the vaso-obliteration areas were significantly smaller than those in the OIR group $(t=16.72, P<0.05)$ and the OIR-GFP group $(t=13.60$, $P<0.05$; Figure 3f). There was no obvious infection, inflammation, toxicity, or other damage related to the intravitreous injection and the dosage administered. Therefore, our results suggested that Ad-S100A4-RNAi transfer significantly reduced oxygen-induced ischaemic RNV and ameliorated hyperoxia-mediated vasoobliteration.

Reduced number of vascular cell nuclei on the vitreal side of the internal limiting membrane in the OIR model

To further confirm the effect of Ad-S100A4-RNAi transfer and determine the degree of oxygen-induced ischaemic RNV, we quantified the number of vascular cell nuclei on the vitreal side of the internal limiting membrane in serial paraffin cross-sections, a characteristic feature of the OIR model. ${ }^{18}$ The results 

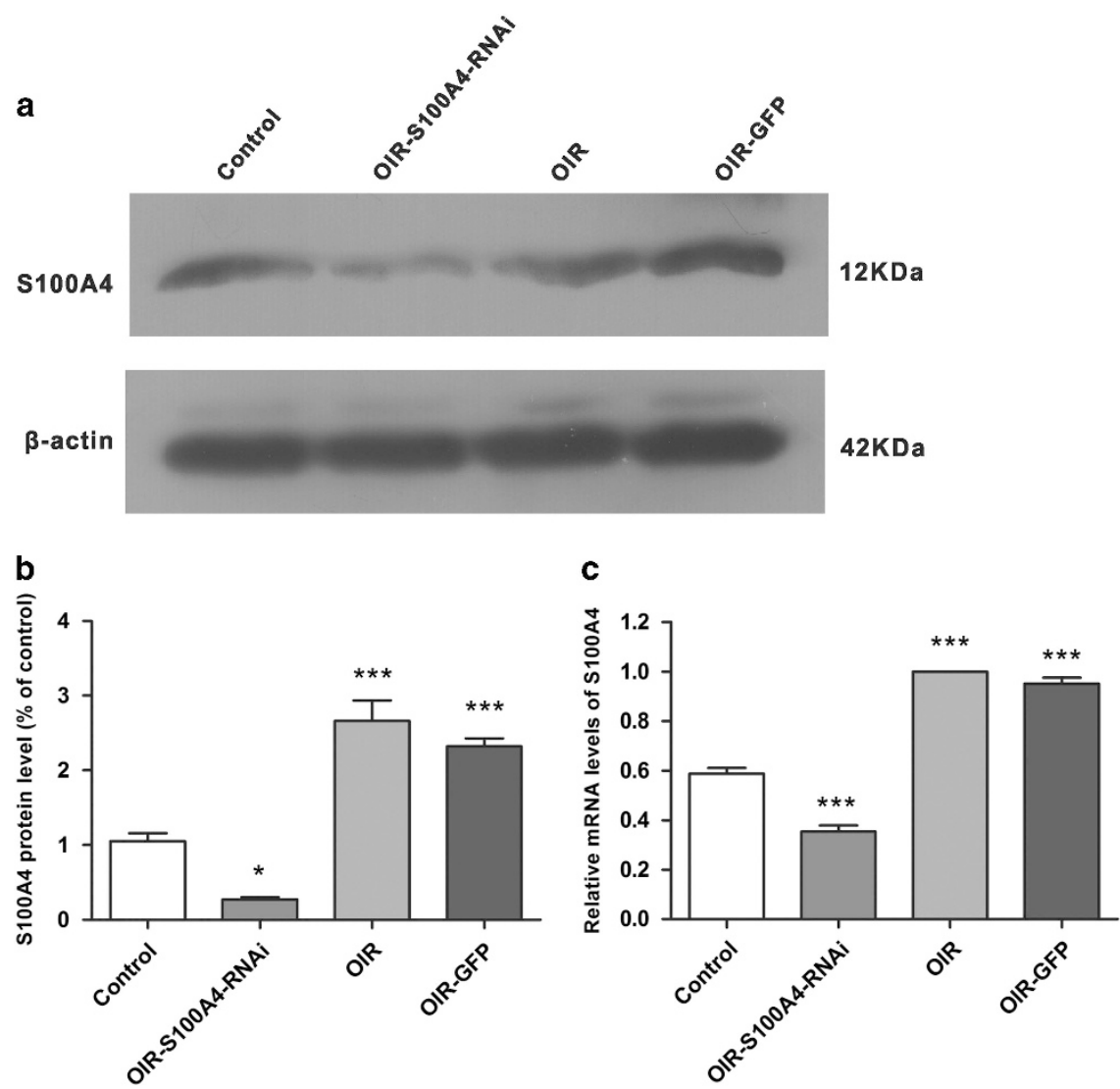

Figure 2 Change in S100A4 expression at the protein and mRNA levels after Ad-S100A4-RNAi transfer into the mouse retina. (a, b) Western blotting confirmed that protein levels of S100A4 were significantly lower in the OIR-S100A4-RNAi group mouse retinas compared with the OIR, OIR-GFP, and control group mouse retinas. The bands were quantified by densitometry and normalised to $\beta$-actin. Data were analysed as the mean \pm SD. OIR-S100A4-RNAi group vs control group, ${ }^{*} P<0.05$; OIR-S100A4-RNAi group vs OIR group, ${ }^{* *} P<0.05$; OIR-S100A4-RNAi group vs OIR-GFP group, ${ }^{* * *} P<0.05$. (c) Real-time PCR analysis further confirmed that mRNA levels of S100A4 in the S100A4 group were significantly lower than those in the control group, OIR group and GFP group mouse retinas. The relative amount of mRNA was normalised to $\beta$-actin. Data are shown as the mean \pm SD. OIR-S100A4-RNAi group vs control group, ${ }^{* * *} P<0.05$; OIR-S100A4-RNAi group vs OIR group, ${ }^{* * *} P<0.05$; OIR-S100A4-RNAi group vs OIR-GFP group, ${ }^{* * * P} P<0.05$.

showed that the number of pre-retinal neovascular cell nuclei in retinas from the OIR-S100A4-RNAi group $(4.25 \pm 2.22)$ were significantly lower than those in the retinas from the OIR group $(53.25 \pm 9.03, t=10.94$, $P<0.05)$ and OIR-GFP group $(51.00 \pm 8.60, t=10.44$, $P<0.05$; Figure $4 a-e)$, which confirmed the antineovascularization effect of Ad-S100A4-RNAi transfer into the retina.

\section{Mechanistic study}

Ad-S100A4-RNAi transfer downregulates BDNF, VEGF, and HIF-1 $\alpha$ protein expression.

We compared the expression levels of BDNF, VEGF, and HIF- $1 \alpha$ in the four groups to investigate the inhibitory mechanism of Ad-S100A4-RNAi transfer in RNV. As shown by western blot analysis, protein levels of BDNF, VEGF, and HIF- $1 \alpha$ in the OIR-S100A4-RNAi group were downregulated compared with those in the OIR group

$$
(t=8.42, t=6.11, t=4.44, P<0.05) \text { and OIR-GFP group }
$$$$
(t=9.05, t=5.28, t=5.82, P<0.05 \text {; Figures } 5 \mathrm{a} \text { and } \mathrm{b}) \text {. }
$$

\section{Ad-S100A4-RNAi transfer downregulates BDNF, VEGF, and HIF-1 $\alpha$ mRNA expression}

To further investigate the above changes, real-time PCR was used to analyse the mRNA levels of BDNF, VEGF, and HIF- $1 \alpha$. The results demonstrated that the mRNA levels of BDNF, VEGF, and HIF-1 $\alpha$ significantly decreased in the OIR-S100A4-RNAi group compared with those in the OIR group $(t=3.05, t=3.16, t=3.50, P<0.05)$ and the OIR-GFP group $(t=3.67, t=2.59, t=2.84, P<0.05$; Figure 5c). In brief, the results suggest that the AdS100A4-RNAi transfer performed in the OIR mouse model may ameliorate RNV by downregulating the protein and mRNA levels of BDNF, VEGF, and HIF-1 $\alpha$. 

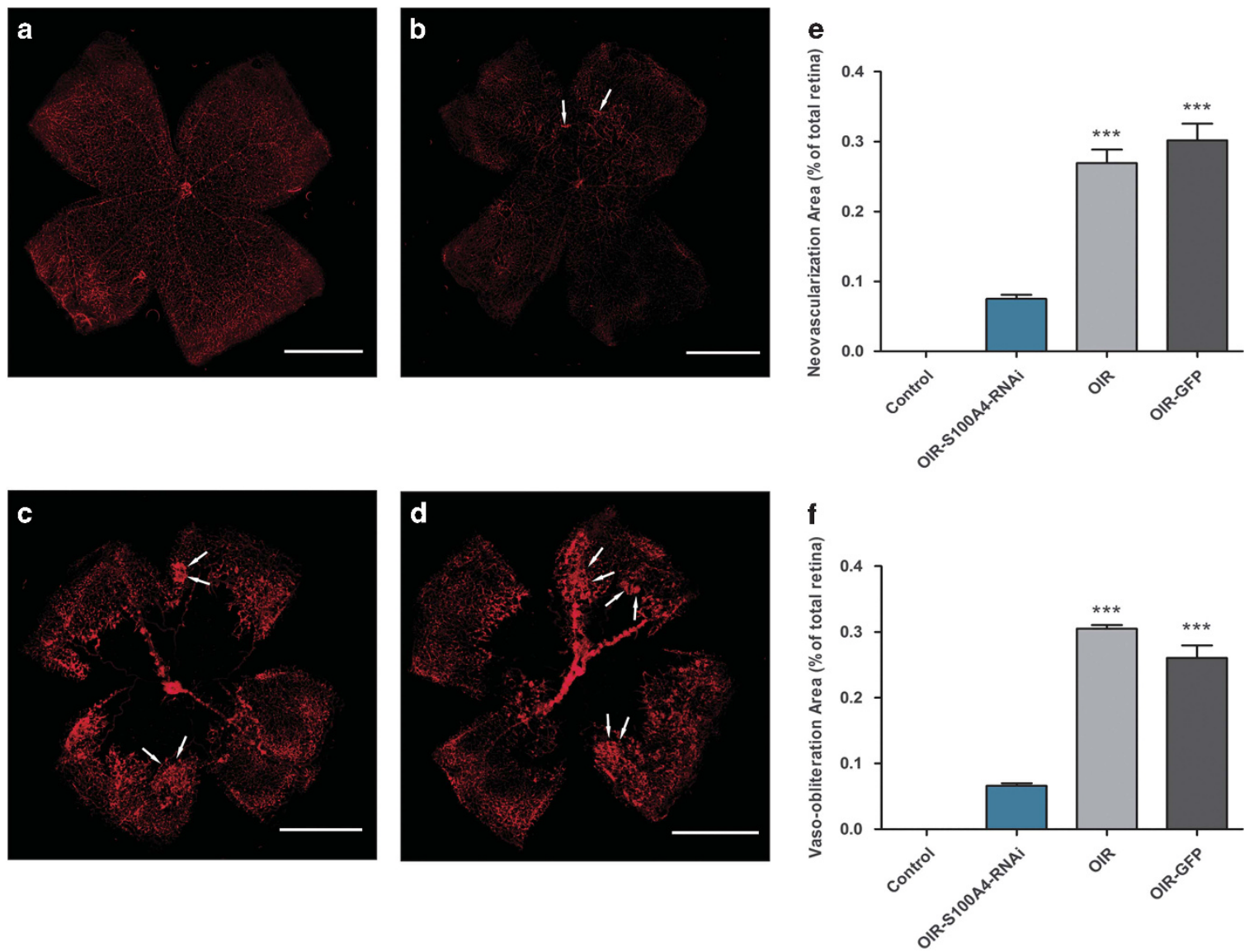

Figure 3 Effects of Ad-S100A4-RNAi transfer on oxygen-induced ischaemic retinal neovascularization. Immunofluorescence staining of vasculature on retinal flat mounts at P17. (a) Control group; (b) OIR-S100A4-RNAi group; (c) OIR group; (d) OIR-GFP group; 40× magnification. The white arrows indicate the neovascular tufts. (e) Quantification of the neovascularization area in the retinas from the OIR-S100A4-RNAi group, OIR group, and OIR-GFP group. Retinal neovascularization was quantified by measuring the ratio of the neovascular tuft area to the total retinal area using Image J 1.48V software (NIH; mean \pm SD). OIR-S100A4-RNAi group vs OIR group, ${ }^{* * *} P<0.05$; OIR-S100A4-RNAi group vs OIR-GFP group, ${ }^{* * *} P<0.05$. (f) Retinal vaso-obliteration areas were measured by calculating the ratio of the non-perfusion area to the total retinal area using Image J 1.48V software (NIH; mean \pm SD). OIR-S100A4-RNAi group vs OIR group, ${ }^{* * *} P<0.05$; OIR-S100A4-RNAi group vs OIR-GFP group, ${ }^{* * *} P<0.05$.

\section{Discussion}

RNV is a serious ophthalmological complication and a major cause of visual impairment; it is observed in ischaemic retinopathies such as proliferative diabetic retinopathy, retinopathy of prematurity, central vein occlusion, and branch retinal vein occlusion. ${ }^{21}$ Recently, new therapies have been developed to target the expression and activity of the pro-angiogenic cytokine VEGF, which, along with its receptors, has emerged as a key biochemical modulator of RNV.22 Previous studies have shown that RNV is tightly associated with an increase in VEGF expression. ${ }^{23}$ Therefore, the identification and therapeutic targeting of signalling pathways involved in the regulation of VEGF in the ischaemic retina could potentially block RNV. In searching for potential targets for RNV treatment, a member of the S100 family, S100A4, attracted our attention.
S100A4, a member of the S100 family of EF-hand calcium-binding proteins, is a pro-angiogenic factor that directly participates in angiogenesis in other tissues, especially in tumour-associated angiogenesis, as well as in endothelial cell migration. ${ }^{10,11,24-29}$ This indicates that S100A4 could act in association with other angiogenic factors to achieve responses both in vitro and in vivo. Endothelial cells of retinal vessels normally have tight junctions that maintain the inner blood-retinal barrier (BRB). ${ }^{30}$ During RNV, new retinal vessels are deficient in endothelial tight junctions, leading to retinal vascular hyperpermeability and inflammation that disrupt normal retinal tissue or function. ${ }^{31}$ In the $\mathrm{BRB}$, the main physical barrier is formed by retinal endothelial cells, which have a key role in the expression of S100A4 during RNV. ${ }^{32}$ As for ocular neovascularization, Changyou Li et al ${ }^{33}$ reported that S100A4 is involved in inflammatory corneal 

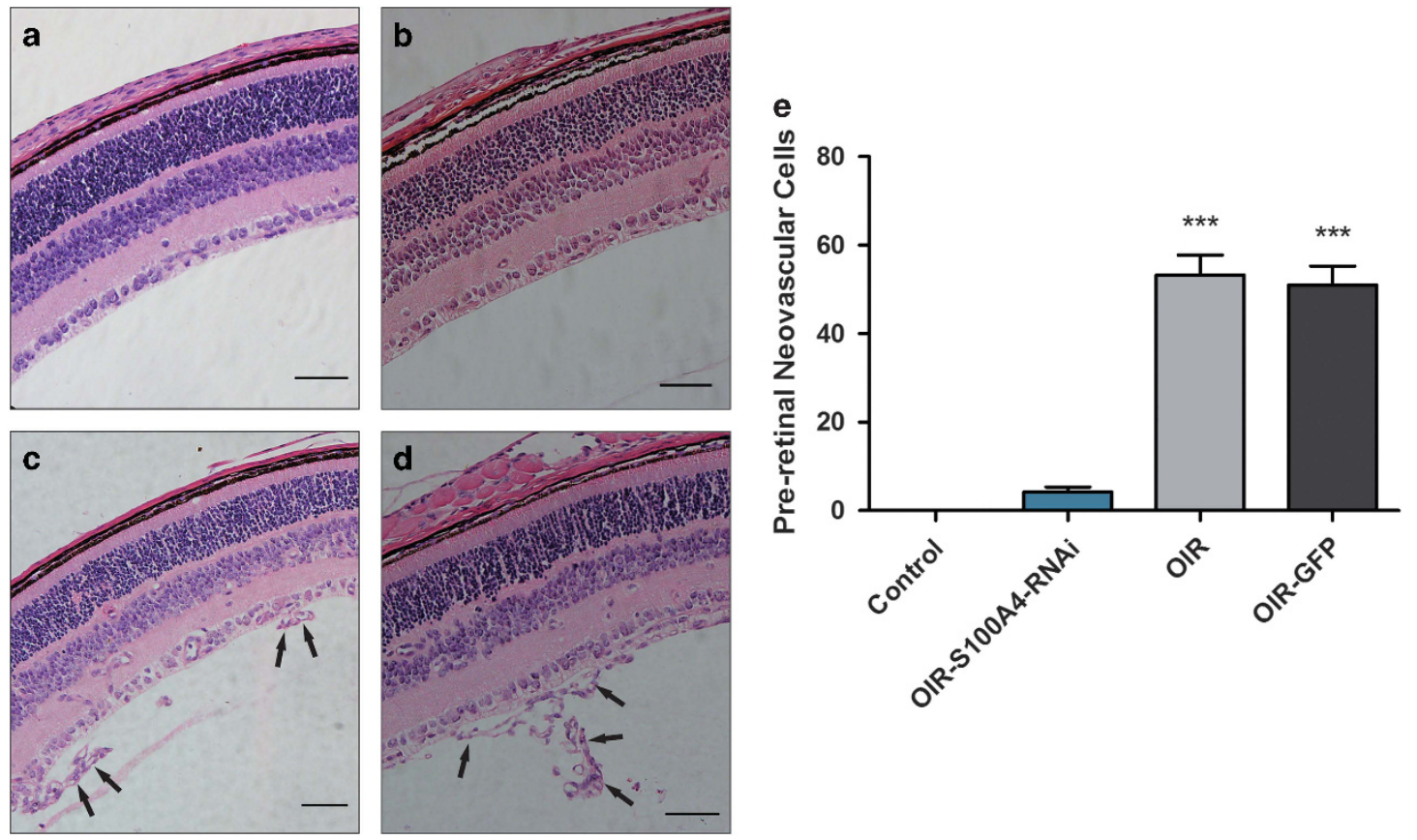

Figure 4 Number of vascular cell nuclei on the vitreal side of the internal limiting membrane in the different groups. The eyeballs from the control group, OIR-S100A4-RNAi group, OIR group, and OIR-GFP group were fixed, sectioned, and stained with H\&E. (a) Control group; (b) OIR-S100A4-RNAi group; (c) OIR group; (d) OIR-GFP group. Arrows indicate pre-retinal neovascularization cells; $200 \times$ magnification. (e) The average numbers of pre-retinal neovascularization cells (mean \pm SD) of the control group, OIR-S100A4-RNAi group, OIR group, and OIR-GFP group were analysed using a one-way ANOVA test. OIR-S100A4-RNAi group vs OIR group, ${ }_{* * *} P<0.05$; OIR-S100A4-RNAi group vs OIR-GFP group, ${ }^{* * *} P<0.05$.

neovascularization. Ahmed et $a^{34}$ found a significant correlation between the vitreous levels of S100A4 and those of the angiogenic biomarker VEGF. In addition, in our preliminary experiment, we found that the protein level of S100A4 was increased in the OIR group compared with the control group. These observations motivated us to examine a possible role for S100A4 in the retina by silencing it. However, whether S100A4 is involved in oxygen-induced ischaemic RNV had not been investigated in previous studies. In the present study, adenoviral-mediated S100A4 RNAI transfer was used to silence the S100A4 gene in the retina, and the results showed that neovascular tufts, vaso-obliteration areas and pre-retinal neovascular cells were all suppressed. These observations indicated that $\mathrm{S} 100 \mathrm{~A} 4$ functions as a pro-angiogenic factor in the RNV process. To figure out the probable mechanism, we evaluated the transcription and translation levels of BDNF, VEGF, and HIF- $1 \alpha$.

As previously described, RNV is tightly associated with an increase in VEGF expression. ${ }^{35-37}$ Because to the identification of VEGF overexpression as a marker for $\mathrm{RNV}$, we examined the expression level of this protein in mouse retinas from four groups and found its expression to be increased in the OIR mouse model and decreased in the OIR mouse model treated with Ad-S100A4-RNAi. This finding was consistent with those of Jose et al, ${ }^{38}$ who reported that the exogenous addition of recombinant S100A4 increased cell migration by acting synergistically with VEGF. Therefore, we speculated that the silencing of the S100A4 gene may reduce VEGF expression at both the protein and mRNA levels. However, VEGF is a downstream factor regulating angiogenesis, which is also subject to upstream regulation. As a newly reported upstream regulator of VEGF, a role for BDNF during angiogenesis has recently emerged.

BDNF is reported to be an endothelial survival factor ${ }^{39}$ that stimulates angiogenesis ${ }^{40}$ and can increase levels of VEGF via HIF- $1 \alpha .{ }^{41}$ In the present study, we evaluated the protein and mRNA levels of BDNF, VEGF, and HIF- $1 \alpha$ to assess whether there is a quantitative change in the three factors before and after Ad-S100A4-RNAi transfer into the OIR mouse retina, which has not yet been studied. We found that the protein and mRNA levels of BDNF, VEGF, and HIF-1 $\alpha$ were increased in the OIR group compared with the control group. According to previous studies, HIF- $1 \alpha$ is a major regulator of VEGF ${ }^{41}$ and can be activated by BDNF. We suggested that BDNF can upregulate VEGF expression via the activation of HIF- $1 \alpha$ and promote RNV, which is consistent with the findings of Lin et al, ${ }^{42}$ who claimed that BDNF increases VEGF expression and enhances angiogenesis in human chondrosarcoma cells. 

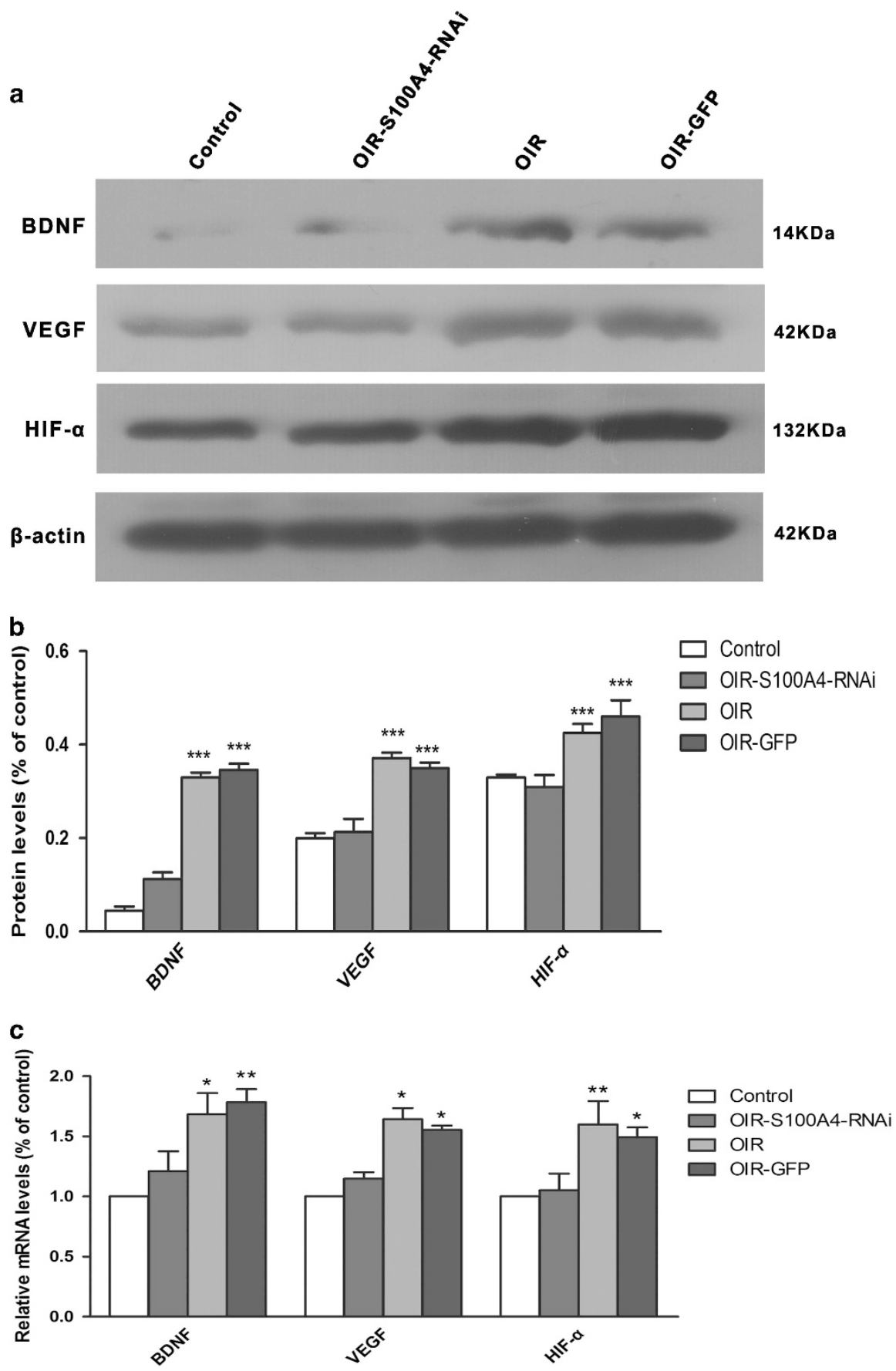

Figure 5 Effects of Ad-S100A4-RNAi transfer on the retinal protein levels of BDNF and VEGF. $(a, b)$ Retinal protein levels of BDNF, VEGF, and HIF-1 $\alpha$ were measured by western blot analysis, which demonstrated that the protein levels of BDNF, VEGF, and HIF-1 $\alpha$ were downregulated in OIR-S100A4-RNAi group than those in OIR and OIR-GFP group. BDNF: OIR-S100A4-RNAi group vs OIR group, ${ }^{* * *} P<0.05$; OIR-S100A4-RNAi group vs OIR-GFP group, ${ }^{* * *} P<0.05$; VEGF: OIR-S100A4-RNAi group vs OIR group, ${ }^{* * * P}<0.05$; OIR-S100A4-RNAi group vs OIR-GFP group, ${ }^{* * *} P<0.05$. HIF-1 $\alpha$ : OIR-S100A4-RNAi group vs OIR group, ${ }^{* * *} P<0.05 ;$ OIR-S100A4-RNAi group vs OIR-GFP, group ***P<0.05. (c) Real-time PCR analysis of BDNF, VEGF, and HIF-1 $\alpha$ expression further confirmed that mRNA levels of BDNF, VEGF, and HIF-1 $\alpha$ significantly decreased in the OIR-S100A4-RNAi group compared with the OIR and OIR-GFP groups. BDNF: OIR-S100A4-RNAi group $v s$ the OIR group, ${ }^{*} P<0.05$; OIR-S100A4-RNAi group vs OIR-GFP group, ${ }^{* *} P<0.05$. VEGF: OIR-S100A4-RNAi group vs OIR group, ${ }^{*} P<0.05$; OIR-S100A4-RNAi group vs OIR-GFP group, ${ }^{*} P<0.05$. HIF-1 $\alpha$ : OIR-S100A4-RNAi group vs OIR group, ${ }^{* *} P<0.05$; OIR-S100A4-RNAi group vs OIR-GFP group, ${ }^{*} P<0.05$. 
After silencing the S100A4 gene in retinas in the OIR mouse model, we found that the protein and mRNA levels of BDNF, VEGF, and HIF-1 $\alpha$ significantly decreased compared with the OIR mouse model without any treatment. This finding may suggest that S100A4 functions as a pro-angiogenic factor and interacts with BDNF, VEGF, and HIF- $1 \alpha$ to promote the RNV process at both the transcriptional and translational levels, which is partially consistent with Ahmed et al, ${ }^{34}$ who claimed that S100A4 is upregulated in proliferative diabetic retinopathy and correlates with markers of angiogenesis.

Taken together, we come to the conclusion that Ad-S100A4-RNAi transfer ameliorates RNV in a mouse model of OIR. The related mechanism involves the silencing of S100A4, which decreases the activation of BDNF and downregulates VEGF expression via HIF- $1 \alpha$. More work should be done to better understand the detailed mechanism of S100A4 function, which could provide new therapeutic targets for the treatment of ocular neovascularization diseases.

\section{Summary}

What was known before
S100A4 promotes angiogenesis in tumour. BDNF/
HIF-1 $\alpha$ /VEGF take part in angiogenesis in tumour.
What this study adds
S100A4 promotes RNV. BDNF/HIF-1 $\alpha$ /VEGF take part in
angiogenesis in RNV.

\section{Conflict of interest}

All authors certify that they have no affiliations with or involvement in any organisation or entity with any financial interest or non-financial interest in the subject matter or materials discussed in this manuscript.

\section{Acknowledgements}

The study was supported by the National Natural Science Foundation of China (grant no. 81271025). The funding organisation had no role in the design or execution of this research. All procedures performed in studies involving human participants were in accordance with the ethical standards of the institution and national research committee and with the 1964 Helsinki declaration and its later amendment or comparable ethical standards.

\section{References}

1 Folkman J, Browder T, Palmblad J. Angiogenesis research: guidelines for translation to clinical application. Thromb Haemost 2001; 86(1): 23-33.
2 Lee P, Wang CC, Adamis AP. Ocular neovascularization: an epidemiologic review. Surv Ophthalmol 1998; 43(3): 245-269.

3 Aiello LP, Northrup JM, Keyt BA, Takagi H, Iwamoto MA. Hypoxic regulation of vascular endothelial growth factor in retinal cells. Arch Ophthalmol 1995; 113(12): 1538-1544.

4 Petrovic V, Bhisitkul RB. Lasers and diabetic retinopathy: the art of gentle destruction. Diabetes Technol Ther 1999; 1(2): 177-187.

5 Sramek CK, Leung LS, Paulus YM, Palanker DV. Therapeutic window of retinal photocoagulation with green (532-nm) and yellow (577-nm) lasers. Ophthalmic Surg Lasers Imaging 2012; 43(4): 341-347.

6 Hernandez C, Simo R. Strategies for blocking angiogenesis in diabetic retinopathy: from basic science to clinical practice. Expert Opin Investig Drugs 2007; 16(8): 1209-1226.

7 Nicholson BP, Schachat AP. A review of clinical trials of anti-VEGF agents for diabetic retinopathy. Graefes Arch Clin Exp Ophthalmol 2010; 248(7): 915-930.

8 Boye K, Maelandsmo GM. S100A4 and metastasis: a small actor playing many roles. Am J Pathol 2010; 176(2): 528-535.

9 de Silva Rudland S, Martin L, Roshanlall C, Winstanley J, Leinster S, Platt-Higgins A et al. Association of S100A4 and osteopontin with specific prognostic factors and survival of patients with minimally invasive breast cancer. Clin Cancer Res 2006; 12(4): 1192-1200.

10 Ambartsumian N, Klingelhofer J, Grigorian M, Christensen C, Kriajevska M, Tulchinsky E et al. The metastasis-associated Mts1(S100A4) protein could act as an angiogenic factor. Oncogene 2001; 20(34): 4685-4695.

11 Schmidt-Hansen B, Ornas D, Grigorian M, Klingelhofer J, Tulchinsky E, Lukanidin E et al. Extracellular S100A4(mts1) stimulates invasive growth of mouse endothelial cells and modulates MMP-13 matrix metalloproteinase activity. Oncogene 2004; 23(32): 5487-5495.

12 Zhang ZJ. Artificial trans-acting small interfering RNA: a tool for plant biology study and crop improvements. Planta 2014; 239(6): 1139-1146.

13 Connor KM, Krah NM, Dennison RJ, Aderman CM, Chen J, Guerin KI et al. Quantification of oxygen-induced retinopathy in the mouse: a model of vessel loss, vessel regrowth and pathological angiogenesis. Nat Protoc 2009; 4(11): 1565-1573.

14 Zhong X, Huang H, Shen J, Zacchigna S, Zentilin L, Giacca M et al. Vascular endothelial growth factor-B gene transfer exacerbates retinal and choroidal neovascularization and vasopermeability without promoting inflammation. Mol Vis 2011; 17: 492-507.

15 Chiu K, Chang RC, So KF. Intravitreous injection for establishing ocular diseases model. J Vis Exp 2007; (8): 313.

16 Fan W, Xing Y, Zhong Y, Chen C, Shen Y. Expression of NMDA receptor subunit 1 in the rat retina. Acta Histochem 2013; 115(1): 42-47.

17 Koriyama Y, Hisano S, Ogai K, Sugitani K, Furukawa A, Kato $\mathrm{S}$. Involvement of neuronal nitric oxide synthase in $\mathrm{N}$-methyl-N-nitrosourea-induced retinal degeneration in mice. J Pharmacol Sci 2015; 127(3): 394-396.

18 Smith LE, Wesolowski E, McLellan A, Kostyk SK, D'Amato R, Sullivan R et al. Oxygen-induced retinopathy in the mouse. Invest Ophthalmol Vis Sci 1994; 35(1): 101-111.

19 Chen YY, Liu SL, Hu DP, Xing YQ, Shen Y. N-methyl-Nnitrosourea-induced retinal degeneration in mice. Exp Eye Res 2014; 121: 102-113. 
20 Livak KJ, Schmittgen TD. Analysis of relative gene expression data using real-time quantitative PCR and the 2(-Delta Delta C(T)) Method. Methods 2001; 25(4): 402-408.

21 Sapieha P, Hamel D, Shao Z, Rivera JC, Zaniolo K, Joyal JS et al. Proliferative retinopathies: angiogenesis that blinds. Int J Biochem Cell Biol 2010; 42(1): 5-12.

22 Penn JS, Madan A, Caldwell RB, Bartoli M, Caldwell RW, Hartnett ME. Vascular endothelial growth factor in eye disease. Prog Retin Eye Res 2008; 27(4): 331-371.

23 Rajappa M, Saxena P, Kaur J. Ocular angiogenesis: mechanisms and recent advances in therapy. Adv Clin Chem 2010; 50: 103-121.

24 Donato R. Intracellular and extracellular roles of S100 proteins. Microsc Res Tech 2003; 60(6): 540-551.

25 Grigorian M, Andresen S, Tulchinsky E, Kriajevska M, Carlberg C, Kruse $\mathrm{C}$ et al. Tumor suppressor p53 protein is a new target for the metastasis-associated Mts1/S100A4 protein: functional consequences of their interaction. J Biol Chem 2001; 276(25): 22699-22708.

26 Yonemura Y, Endou Y, Kimura K, Fushida S, Bandou E, Taniguchi $\mathrm{K}$ et al. Inverse expression of S100A4 and E-cadherin is associated with metastatic potential in gastric cancer. Clin Cancer Res 2000; 6(11): 4234-4242.

27 Takenaga K, Nakanishi H, Wada K, Suzuki M, Matsuzaki O, Matsuura A et al. Increased expression of S100A4, a metastasisassociated gene, in human colorectal adenocarcinomas. Clin Cancer Res 1997; 3(12 Pt 1): 2309-2316.

28 Rudland PS, Platt-Higgins A, Renshaw C, West CR, Winstanley JH, Robertson L et al. Prognostic significance of the metastasis-inducing protein S100A4 (p9Ka) in human breast cancer. Cancer Res 2000; 60(6): 1595-1603.

29 Rosty C, Ueki T, Argani P, Jansen M, Yeo CJ, Cameron JL et al. Overexpression of S100A4 in pancreatic ductal adenocarcinomas is associated with poor differentiation and DNA hypomethylation. Am J Pathol 2002; 160(1): 45-50.

30 Kim J, Kim CS, Lee YM, Jo K, Shin SD, Kim JS. Methylglyoxal induces hyperpermeability of the bloodretinal barrier via the loss of tight junction proteins and the activation of matrix metalloproteinases. Graefes Arch Clin Exp Ophthalmol 2012; 250(5): 691-697.

31 Peng S, Gan G, Rao VS, Adelman RA, Rizzolo LJ. Effects of proinflammatory cytokines on the claudin-19 rich tight junctions of human retinal pigment epithelium. Invest Ophthalmol Vis Sci 2012; 53(8): 5016-5028.

32 Ochiya T, Takenaga K, Endo H. Silencing of S100A4, a metastasis-associated protein, in endothelial cells inhibits tumor angiogenesis and growth. Angiogenesis 2014; 17(1): $17-26$.

33 Li C, Zhang F, Wang Y. S100A proteins in the pathogenesis of experimental corneal neovascularization. Mol Vis 2010; 16: 2225-2235.

34 Abu El-Asrar AM, Nawaz MI, De Hertogh G, Alam K, Siddiquei MM, Van den Eynde $\mathrm{K}$ et al. S100A4 is upregulated in proliferative diabetic retinopathy and correlates with markers of angiogenesis and fibrogenesis. Mol Vis 2014; 20: 1209-1224.

35 Bai Y, Ma JX, Guo J, Wang J, Zhu M, Chen Y et al. Muller cell-derived VEGF is a significant contributor to retinal neovascularization. J Pathol 2009; 219(4): 446-454.

36 Jo DH, Kim S, Kim D, Kim JH, Jon S, Kim JH. VEGF-binding aptides and the inhibition of choroidal and retinal neovascularization. Biomaterials 2014; 35(9): 3052-3059.

37 Rodrigues M, Xin X, Jee K, Babapoor-Farrokhran S, Kashiwabuchi F, Ma T et al. VEGF secreted by hypoxic Muller cells induces MMP-2 expression and activity in endothelial cells to promote retinal neovascularization in proliferative diabetic retinopathy. Diabetes 2013; 62(11): 3863-3873.

38 Hernandez JL, Padilla L, Dakhel S, Coll T, Hervas R, Adan J et al. Therapeutic targeting of tumor growth and angiogenesis with a novel anti-S100A4 monoclonal antibody. PLoS One 2013; 8(9): e72480.

39 Donovan MJ, Lin MI, Wiegn P, Ringstedt T, Kraemer R, Hahn $\mathrm{R}$ et al. Brain derived neurotrophic factor is an endothelial cell survival factor required for intramyocardial vessel stabilization. Development 2000; 127(21): 4531-4540.

40 Kermani P, Rafii D, Jin DK, Whitlock P, Schaffer W, Chiang A et al. Neurotrophins promote revascularization by local recruitment of TrkB+ endothelial cells and systemic mobilization of hematopoietic progenitors. J Clin Invest 2005; 115(3): 653-663.

41 Nakamura K, Martin KC, Jackson JK, Beppu K, Woo CW, Thiele CJ. Brain-derived neurotrophic factor activation of TrkB induces vascular endothelial growth factor expression via hypoxia-inducible factor-1alpha in neuroblastoma cells. Cancer Res 2006; 66(8): 4249-4255.

42 Lin CY, Hung SY, Chen HT, Tsou HK, Fong YC, Wang SW et al. Brain-derived neurotrophic factor increases vascular endothelial growth factor expression and enhances angiogenesis in human chondrosarcoma cells. Biochem Pharmacol 2014; 91(4): 522-533. 